\title{
CIENCIAMATRIA
}

Revista Interdisciplinaria de Humanidades, Educación, Ciencia y Tecnología

Año VIII. Vol. VIII. №14. Enero - Junio. 2022

Hecho el depósito de ley: pp201602FA4721

ISSN-L: 2542-3029; ISSN: 2610-802X

Universidad Nacional Experimental Francisco de Miranda (UNEFM). Santa Ana de Coro. Venezuela

Luís Antonio Visurraga-Camargo

DOI $10.35381 / \mathrm{cm} \cdot v 8 \mathrm{i} 14.636$

\section{Auditoría de gestión y control gubernamental en el sector público. Caso: Junín- Perú}

\section{Performance audit and governmental control in the public sector. Case: Junín-} Peru

\author{
Luís Antonio Visurraga-Camargo \\ visurragacamargol@gmail.com \\ Universidad Nacional Federico Villarreal, Lima \\ Perú \\ https://orcid.org/0000-0002-0429-9017
}

Recibido: 15 de septiembre 2021

Revisado: 10 de noviembre 2021

Aprobado: 15 de diciembre 2021

Publicado: 01 de enero 2022 
CIENCIAMATRIA

Revista Interdisciplinaria de Humanidades, Educación, Ciencia y Tecnología

Año VIII. Vol. VIII. N¹4. Enero - Junio. 2022

Hecho el depósito de ley: pp201602FA4721

ISSN-L: 2542-3029; ISSN: 2610-802X

Universidad Nacional Experimental Francisco de Miranda (UNEFM). Santa Ana de Coro. Venezuela

Luís Antonio Visurraga-Camargo

\title{
RESUMEN
}

El objetivo general de la investigación fue analizar la auditoría de gestión y control gubernamental en el sector público. Caso: Junín - Perú. Se desarrolló mediante la el enfoque cuantitativo, manejando la recolección y análisis documental-biográfica, lo que consistió en organizar y analizar el objeto de estudio. Mediante la recolección de datos y uso de estadística. Se distinguieron las variables de estudio referidas a la auditoria de gestión y control gubernamental, con sus respectivas dimensiones. La población estuvo constituida de 338, entre Autoridades (Gobernador Regional, Alcaldes Provinciales y Distritales) y Funcionarios. Cuya muestra considerada fue de 180. A los que se les aplicó un cuestionario. Se concluye que existe una relación directa y significativa entre la auditoria de gestión y el control gubernamental en el sector público de la Región Junín. Se sugiere a las instituciones públicas y sus funcionarios, en coordinación con el Órgano de control interno supervisar y vigilar.

Descriptores: Auditoria de gestión; control de gestión; sector público; política gubernamental; función pública. (Tesauro UNESCO).

\begin{abstract}
The general objective of the research was to analyze performance auditing and governmental control in the public sector. Case: Junín - Peru. It was developed through the quantitative approach, using documentary-biographic collection and analysis, which consisted of organizing and analyzing the object of study. Through data collection and the use of statistics. The study variables referred to performance auditing and governmental control, with their respective dimensions, were distinguished. The population consisted of 338, including authorities (Regional Governor, Provincial and District Mayors) and civil servants. The sample considered was 180 , to whom a questionnaire was applied. It is concluded that there is a direct and significant relationship between performance audit and governmental control in the public sector of the Junin Region. It is suggested to the public institutions and their officials, in coordination with the internal control organ, to supervise and monitor.
\end{abstract}

Descriptors: Performance auditing; management control; public sector; government policy; public function. (UNESCO Thesaurus). 


\section{CIENCIAMATRIA}

Revista Interdisciplinaria de Humanidades, Educación, Ciencia y Tecnología

Año VIII. Vol. VIII. №14. Enero - Junio. 2022

Hecho el depósito de ley: pp201602FA4721

ISSN-L: 2542-3029; ISSN: 2610-802X

Universidad Nacional Experimental Francisco de Miranda (UNEFM). Santa Ana de Coro. Venezuela

Luís Antonio Visurraga-Camargo

\section{INTRODUCCIÓN}

Los Gobiernos Regionales empiezan a funcionar a partir del $1^{\circ}$ de enero del año 2003, iniciándose el proceso de descentralización, por cuanto muchas funciones y responsabilidades que eran competencia de los Ministerios (Gobierno Central) fueron transferidas a los Gobiernos Regionales, especialmente la ejecución de proyectos de envergadura en cada Región, asignándoles presupuestos altos que nunca habían manejado desde S/ 100,000 soles hasta S/ 400'000,000 de soles, lo que permitió que las Autoridades y Funcionarios Públicos, utilicen estos recursos sin respetar las normas y procedimientos establecidos en los diferentes sistemas administrativos, trayendo como consecuencia que muchos Presidentes y Gobernadores Regionales así como Funcionarios, fueran denunciados y sentenciados y hoy vienen cumpliendo penas privativas de libertad en los diferentes centros penitenciarios del país.

En este contexto, en la actualidad se puede observar como todas las instituciones de la administración pública de nuestro país se encuentra convulsionado por los actos de corrupción, no solo en el ámbito judicial sino también en el ámbito ejecutivo y esto es por la falta de control, el mismo que debe ser ejercido como un control previo por las Autoridades, Funcionarios y servidores públicos, y el control posterior por la Contraloría General de la Republica y los Órganos de Control Institucional de todas las Instituciones Públicas. Las Autoridades, Funcionarios y servidores públicos no poseen el conocimiento de las normas establecidas para ejecutar una Auditoría de Gestión o Auditoría Administrativa en las Instituciones del Estado, así como también del Control Gubernamental y mucho menos no se entiende y comprende quienes tienen la responsabilidad de implementar y fortalecer el Sistema de control interno y externo al interior de una entidad u organización pública. Al respeto, Cabana et al. (2020) plantea:

Hoy más que nunca se le da relevancia al control de las instituciones públicas, porque es fundamental que funcione bien para facilitar la efectividad del gobierno institucional. Los controles se implantan con el fin de detectar, en el plazo deseado, cualquier desviación respecto a los objetivos establecidos por la entidad y de limitar las sorpresas. (p.104) 


\section{CIENCIAMATRIA}

Revista Interdisciplinaria de Humanidades, Educación, Ciencia y Tecnología

Año VIII. Vol. VIII. №14. Enero - Junio. 2022

Hecho el depósito de ley: pp201602FA4721

ISSN-L: 2542-3029; ISSN: 2610-802X

Universidad Nacional Experimental Francisco de Miranda (UNEFM). Santa Ana de Coro. Venezuela

Luís Antonio Visurraga-Camargo

Sin embargo, la doble funcionalidad entre los gobiernos regionales, los municipios provinciales y distritales, con las unidades ejecutivas del gobierno central y los programas sociales llevó a un doble uso de los recursos económicos, materiales y económicos, lo que perjudicó al erario nacional. En tal sentido, el fundamento teórico de la investigación, está referido a la Auditoría de Gestión, sustentado por diferentes investigadores, referidos a sus dimensiones como la efectividad, la eficiencia y la economía. Asimismo, el Control Gubernamental está referido a sus dimensiones como la supervisión, vigilancia, verificación y resultados.

Se muestra a continuación los antecedentes que han orientado el desarrollo investigativo:

Cuadro 1.

\section{Antecedentes.}

\begin{tabular}{|l|l|l|}
\hline \multicolumn{1}{|c|}{ Autor(es) } & \multicolumn{1}{|c|}{ Titulo } & \multicolumn{1}{c|}{ Desarrollo } \\
\hline $\begin{array}{l}\text { Santa María, G.; } \\
\text { Cárdenas, M. y Vega } \\
\text { Rivera (2016). }\end{array}$ & $\begin{array}{l}\text { La Auditoria de Gestión, } \\
\text { una Herramienta Necesaria } \\
\text { para la Economía }\end{array}$ & $\begin{array}{l}\text { Este trabajo tiene como } \\
\text { objetivo describir algunos } \\
\text { posicionamientos teóricos y } \\
\text { diferentes ámbitos de } \\
\text { aplicación de la auditoría de } \\
\text { gestión como una } \\
\text { herramienta eficaz para las } \\
\text { organizaciones. } \\
\text { Concluyeron: a. La } \\
\text { auditoría de gestión es una } \\
\text { herramienta aplicable en } \\
\text { cualquier tipo de entidad, } \\
\text { puede ser pública o } \\
\text { privada, y contribuye a la } \\
\text { administración en la } \\
\text { evaluación de un sistema, } \\
\text { proceso o subproceso. } \\
\text { Genere resultados que } \\
\text { faciliten la disminución de } \\
\text { costos y la simplificación de } \\
\text { tareas innecesarias o o }\end{array}$ \\
\hline
\end{tabular}


CIENCIAMATRIA

Revista Interdisciplinaria de Humanidades, Educación, Ciencia y Tecnología

Año VIII. Vol. VIII. N¹4. Enero - Junio. 2022

Hecho el depósito de ley: pp201602FA4721

ISSN-L: 2542-3029; ISSN: 2610-802X

Universidad Nacional Experimental Francisco de Miranda (UNEFM). Santa Ana de Coro. Venezuela

Luís Antonio Visurraga-Camargo

\begin{tabular}{|c|c|c|}
\hline & & irrelevantes. \\
\hline Portal, J. (2016). & $\begin{array}{lcr}\text { Control } & \text { Interno } & \text { e } \\
\text { integridad: } & \text { elementos } \\
\text { necesarios } & \text { para la } \\
\text { Gobernanza } & \text { Pública. }\end{array}$ & $\begin{array}{l}\text { El concepto de gobernanza } \\
\text { ha dado lugar al desarrollo } \\
\text { de diversos esquemas de } \\
\text { gestión los cuales están } \\
\text { centrados en el éxito de las } \\
\text { organizaciones en términos } \\
\text { de eficacia, eficiencia, } \\
\text { transparencia y legalidad. }\end{array}$ \\
\hline Calle (2015). & $\begin{array}{l}\text { Auditoría de gestión como } \\
\text { herramienta de } \\
\text { mejoramiento continuo en } \\
\text { la empresa municipal de } \\
\text { agua potable, alcantarillado } \\
\text { y saneamiento ambiental } \\
\text { del cantón azogues } \\
\text { (Emapal), periodo } 2013 \text {. }\end{array}$ & $\begin{array}{l}\text { Falta de un código de ética, } \\
\text { provoco que las actividades } \\
\text { se desempeñen bajo } \\
\text { criterios personales. No } \\
\text { existe un mecanismo ni un } \\
\text { plan de mitigación de } \\
\text { riesgos que permita } \\
\text { identificar, analizar y } \\
\text { evaluar los riesgos. }\end{array}$ \\
\hline Flores (2014). & $\begin{array}{l}\text { Planeamiento y estrategias } \\
\text { en la auditoría de gestión } \\
\text { en las instituciones } \\
\text { públicas del sector energía } \\
\text { y minas. }\end{array}$ & $\begin{array}{l}\text { El planeamiento eficiente, y } \\
\text { estrategias en la auditoría } \\
\text { en la Instituciones Públicas } \\
\text { del Sector Energía y Minas } \\
\text { influye significativamente } \\
\text { en la eficacia de la } \\
\text { evaluación de gestión y } \\
\text { control de recursos y bienes } \\
\text { públicos, la toma de } \\
\text { decisiones gerenciales y el } \\
\text { cumplimiento de metas y } \\
\text { objetivos institucionales. }\end{array}$ \\
\hline Aroca (2016) & $\begin{array}{l}\text { La Auditoría Interna y su } \\
\text { Incidencia en la Gestión De } \\
\text { Empresa De Transportes } \\
\text { Guzmán S.A. de la Ciudad } \\
\text { de Trujillo. }\end{array}$ & $\begin{array}{l}\text { La contrastación y el } \\
\text { análisis de la información } \\
\text { recolectada y la aplicación } \\
\text { de los instrumentos para la } \\
\text { presente investigación } \\
\text { permitieron determinar que } \\
\text { la Auditoría Interna, incide } \\
\text { favorablemente en la } \\
\text { Gestión de Empresa de } \\
\text { Transportes Guzmán S.A. } \\
\text { de la ciudad de Trujillo. }\end{array}$ \\
\hline
\end{tabular}


Elaboración: El autor.

Por lo tanto, Zambrano-Zambrano y Armada-Trabas (2018) menciona que la auditoría de gestión:

Es una necesidad social establecer procedimientos de auditoría de gestión como actividad con basamento científico que permitan medir el cumplimiento de la misión, la consecución de los objetivos institucionales y la utilización de los fondos públicos recibidos con eficiencia, transparencia, oportunidad y según el marco legal establecido por la Contraloría General del Estado. (p.17)

En este sentido, el concepto de auditoría de gestión, con su preocupación de control operativo, es el puente, y a veces el catalizador entre una auditoría financiera tradicional, y un método de servicios administrativos para solucionar el problema de un tiempo. Constituye un ingrediente necesario en la plena implantación de la auditoría integral. Los programas de auditoría de gestión específicos para cada área funcional mayor, proporciona al auditor una guía con respecto a los tipos de controles, condiciones y circunstancias que probablemente logre encontrar.

Por otro lado, en el país, el control gubernamental se encuentra normado en la Ley Orgánica del Sistema Nacional de Control y de la Contraloría General de la República Ley $\mathrm{N}^{\circ} 27785$, donde se señala que:

El Control Gubernamental consiste en la supervisión, vigilancia y verificación de los actos y resultados de la gestión pública, en atención al grado de eficiencia, eficacia, transparencia y economía en el uso y destino de los recursos y bienes del estado, así como del cumplimiento de las normas legales y de los lineamientos de política y planes de acción, evaluando los sistemas de administración, gerencia y control, con fines de su mejoramiento a través de la adopción de acciones preventivas y correctivas pertinentes. 


\section{CIENCIAMATRIA \\ Revista Interdisciplinaria de Humanidades, Educación, Ciencia y Tecnología \\ Año VIII. Vol. VIII. N¹4. Enero - Junio. 2022 \\ Hecho el depósito de ley: pp201602FA4721 \\ ISSN-L: 2542-3029; ISSN: 2610-802X \\ Universidad Nacional Experimental Francisco de Miranda (UNEFM). Santa Ana de Coro. Venezuela}

Luís Antonio Visurraga-Camargo

El control gubernamental es interno y externo. Su desarrollo constituye un proceso integral y permanente. El control interno se refiere a las acciones que desarrolla la propia entidad pública con la finalidad de que la gestión de sus recursos, bienes y operaciones se efectúe correcta y eficientemente. El control externo es entendido como el conjunto de políticas, normas, métodos y procedimientos técnicos que compete aplicar a la Contraloría

General u otro órgano del Sistema Nacional de Control por encargo o designación de esta, con el objeto de supervisar, vigilar y verificar la gestión, la captación y el uso de los recursos y bienes del Estado. La corrupción es un fenómeno que ocasiona devastadoras consecuencias para la administración pública, pues socava su credibilidad y obstruye el cumplimiento de los fines que persiguen sus órganos e instituciones. (Madrid Valerio y Palomino Ramírez, 2020, p.214).

De acuerdo a los planteamientos formulados el objetivo general de la investigación es analizar la auditoría de gestión y control gubernamental en el sector público. Caso: Junín - Perú.

\section{METODOLOGÍA}

En el presente trabajo se desarrolló mediante la metodología, basada en el enfoque cuantitativo, manejando la recolección y análisis documental-bibliográfica, (Hernández Sampieri, Fernández Collado, y Baptista Lucio, 2014), lo cual permitió organizar un análisis del objeto de estudio. Para la investigación cuantitativa se maneja la recolección y el análisis de datos, que mediante el uso de la estadística, permite establecer información confiable del comportamiento de una población específica. Se realizó la distinción de las variables de estudios referidas a la auditoria de gestión, cuyas dimensiones fueron efectividad, deficiencia y economía, así mismos control gubernamental y supervisión de gestión. La población estaba constituida de 338, entre Autoridades (Gobernador Regional, Alcaldes Provinciales y Distritales) y Funcionarios y 


\section{CIENCIAMATRIA}

Revista Interdisciplinaria de Humanidades, Educación, Ciencia y Tecnología

Año VIII. Vol. VIII. №14. Enero - Junio. 2022

Hecho el depósito de ley: pp201602FA4721

ISSN-L: 2542-3029; ISSN: 2610-802X

Universidad Nacional Experimental Francisco de Miranda (UNEFM). Santa Ana de Coro. Venezuela

Luís Antonio Visurraga-Camargo

la Muestra utilizada fueron de 180 personas entre Autoridades y Funcionarios. La técnica de muestreo será aleatorio simple; y se aplicará la técnica de la encuesta y el análisis documental. Se aplicó los cuestionarios a los funcionarios y trabajadores del sector público de la Región Junín, se realizará en la sede de cada institución pública, en un solo momento de persona a persona, por Unidades Orgánicas.

\section{RESULTADOS}

Se muestran a continuación los resultados obtenidos de la encuetas aplicada a los funcionarios y trabajadores del sector público de la Región Junín.

Tabla 1.

Auditoria de gestión.

\begin{tabular}{llrrrr}
\hline & & & \multicolumn{2}{c}{$\begin{array}{c}\text { Porcentaje } \\
\text { válido }\end{array}$} & $\begin{array}{c}\text { Porcentaje } \\
\text { acumulado }\end{array}$ \\
\hline Válido & NUNCA & 18 & 10.0 & 10.0 & 10.0 \\
& CASI NUNCA & 11 & 6.1 & 6.1 & 16.1 \\
& A VECES & 66 & 36.7 & 36.7 & 52.8 \\
& CASI SIEMPRE & 49 & 27.2 & 27.2 & 80.0 \\
& SIEMPRE & 36 & 20.0 & 20.0 & 100.0 \\
& Total & 180 & 100.0 & 100.0 & \\
\hline \hline
\end{tabular}

Fuente: Encuesta.

De los 180 encuestados, según la tabla № 01, un $10.00 \%$ (15) y $6.1 \%$ (11) manifiesta que la auditoria de la gestión en el sector público de la Región es nada efectiva (Nunca) y (casi nunca) respectivamente, tanto que un $36.7 \%$ (66) de los encuestados manifiesta un grado de efectividad intermedia (a veces), en contraste con un $27.2 \%$ (49) y un 20.0 $\%(36)$ que manifiestan una alta y muy alta efectividad respectivamente (casi siempre) y (siempre). 


\section{CIENCIAMATRIA}

Revista Interdisciplinaria de Humanidades, Educación, Ciencia y Tecnología

Año VIII. Vol. VIII. N¹4. Enero - Junio. 2022

Hecho el depósito de ley: pp201602FA4721

ISSN-L: 2542-3029; ISSN: 2610-802X

Universidad Nacional Experimental Francisco de Miranda (UNEFM). Santa Ana de Coro. Venezuela

Luís Antonio Visurraga-Camargo

\section{Tabla 2}

Efectividad.

\begin{tabular}{|c|c|c|c|c|c|}
\hline & & Frecuencia & Porcentaje & $\begin{array}{c}\text { Porcentaje } \\
\text { válido }\end{array}$ & $\begin{array}{l}\text { Porcentaje } \\
\text { acumulado }\end{array}$ \\
\hline \multirow[t]{6}{*}{ Válido } & NUNCA & 7 & 3.9 & 3.9 & 3.9 \\
\hline & CASI NUNCA & 14 & 7.8 & 7.8 & 11.7 \\
\hline & A VECES & 66 & 36.7 & 36.7 & 48.3 \\
\hline & CASI SIEMPRE & 60 & 33.3 & 33.3 & 81.7 \\
\hline & SIEMPRE & 33 & 18.3 & 18.3 & 100.0 \\
\hline & Total & 180 & 100.0 & 100.0 & \\
\hline
\end{tabular}

Fuente: Encuesta.

De los 180 encuestados, según la tabla № 02, un $3.89 \%$ (7) y un $7.78 \%$ (14) manifiesta que la efectividad de la gestión en el sector público de la Región es nula (Nunca) y (casi nunca) respectivamente, tanto que un $18.33 \%$ (33) de los encuestados manifiesta un grado de efectividad intermedia (a veces), en contraste con un $33.33 \%$ (60) y un 36.67 $\%(66)$ que manifiestan una alta y muy alta efectividad de la gestión (casi siempre) y (a veces).

\section{Tabla 3.}

Eficiencia

\begin{tabular}{llrrrr}
\hline & & & \multicolumn{2}{c}{$\begin{array}{c}\text { Porcentaje } \\
\text { válido }\end{array}$} & $\begin{array}{c}\text { Porcentaje } \\
\text { acumulado }\end{array}$ \\
\hline Válido & NUNCA & 15 & 8.3 & 8.3 & 8.3 \\
& CASI NUNCA & 30 & 16.7 & 16.7 & 25.0 \\
& A VECES & 50 & 27.8 & 27.8 & 52.8 \\
CASI SIEMPRE & 69 & 38.3 & 38.3 & 91.1 \\
& SIEMPRE & 16 & 8.9 & 8.9 & 100.0 \\
Total & 180 & 100.0 & 100.0 & \\
\hline
\end{tabular}

Fuente: Encuesta. 


\section{CIENCIAMATRIA}

Revista Interdisciplinaria de Humanidades, Educación, Ciencia y Tecnología

Año VIII. Vol. VIII. N¹4. Enero - Junio. 2022

Hecho el depósito de ley: pp201602FA4721

ISSN-L: 2542-3029; ISSN: 2610-802X

Universidad Nacional Experimental Francisco de Miranda (UNEFM). Santa Ana de Coro. Venezuela

Luís Antonio Visurraga-Camargo

De los 180 encuestados, según la tabla № 03, un $8.33 \%$ (15) y $8.89 \%$ (30) manifiesta que la eficiencia de la gestión en el sector público de la Región es nula (Nunca) y (siempre) respectivamente, tanto que un $16.67 \%$ (30) de los encuestados manifiesta un grado de eficiencia intermedia (casi nunca), en contraste con un $27.78 \%$ (59) y un $38.33 \%(69)$ que manifiestan una alta y muy alta eficiencia de la gestión respectivamente (a veces) y (casi siempre).

Tabla 4.

Economía.

\begin{tabular}{llrrrr}
\hline & & & \multicolumn{2}{c}{$\begin{array}{c}\text { Porcentaje } \\
\text { válido }\end{array}$} & $\begin{array}{c}\text { Porcentaje } \\
\text { acumulado }\end{array}$ \\
\hline Válido & NUNCA & 11 & 6.1 & 6.1 & 6.1 \\
& CASI NUNCA & 36 & 20.0 & 20.0 & 26.1 \\
& A VECES & 62 & 34.4 & 34.4 & 60.6 \\
& CASI SIEMPRE & 55 & 30.6 & 30.6 & 91.1 \\
& SIEMPRE & 16 & 8.9 & 8.9 & 100.0 \\
& Total & 180 & 100.0 & 100.0 & \\
\hline
\end{tabular}

Fuente: Encuesta.

De los 180 encuestados, según la tabla № 04, un $6.11 \%$ (11) y $8.89 \%$ (16) manifiesta que la eficiencia de la gestión en el sector público de la Región es nula (Nunca) y (siempre) respectivamente, tanto que un $20.00 \%$ (36) de los encuestados manifiesta un grado de eficiencia intermedia (casi nunca), en contraste con un $30.56 \%$ (55) y un 34.44 $\%(62)$ que manifiestan una alta y muy alta eficiencia de la gestión (casi siempre) y (a veces). 


\section{CIENCIAMATRIA}

Revista Interdisciplinaria de Humanidades, Educación, Ciencia y Tecnología

Año VIII. Vol. VIII. N¹4. Enero - Junio. 2022

Hecho el depósito de ley: pp201602FA4721

ISSN-L: 2542-3029; ISSN: 2610-802X

Universidad Nacional Experimental Francisco de Miranda (UNEFM). Santa Ana de Coro. Venezuela

Luís Antonio Visurraga-Camargo

\section{Tabla 5.}

Control gubernamental.

\begin{tabular}{llrrrr}
\hline & & & & \multicolumn{2}{c}{$\begin{array}{c}\text { Porcentaje } \\
\text { válido }\end{array}$} \\
\hline Válido & NUNCA & Precuencia & \multicolumn{1}{c}{$\begin{array}{c}\text { Porcentaje } \\
\text { acumulado }\end{array}$} \\
& A VECES & 20 & 11.1 & 11.1 & 11.1 \\
& CASI SIEMPRE & 65 & 36.1 & 36.1 & 47.2 \\
& SIEMPRE & 40 & 22.2 & 22.2 & 69.4 \\
& Total & 55 & 30.6 & 30.6 & 100.0 \\
\hline
\end{tabular}

Fuente: Encuesta.

De los 180 encuestados, según la tabla № 05, un $11.1 \%$ (20) manifiesta que el control gubernamental en el sector público de la Región Junín es nula (Nunca ) y un 22.2\% (40) de los encuestados manifiestan el control gubernamental es casi nula (casi siempre), en tanto que un $30.6 \%$ (55) de los encuestados manifiestan que el control gubernamental es intermedia (siempre), y un $36.1 \%(65)$ que manifiestan que el control gubernamental es alta (a veces).

\section{Tabla 6.}

Supervisión de la Gestión.

\begin{tabular}{llrrrr}
\hline & & & \multicolumn{2}{c}{$\begin{array}{c}\text { Porcentaje } \\
\text { válido }\end{array}$} & $\begin{array}{c}\text { Porcentaje } \\
\text { acumulado }\end{array}$ \\
\hline Válido & NUNCA & 21 & 11.7 & 11.7 & 11.7 \\
& CASI NUNCA & 31 & 17.2 & 17.2 & 28.9 \\
& A VECES & 58 & 32.2 & 32.2 & 61.1 \\
& CASI SIEMPRE & 67 & 37.2 & 37.2 & 98.3 \\
& SIEMPRE & 3 & 1.7 & 1.7 & 100.0 \\
& Total & 180 & 100.0 & 100.0 & \\
\hline
\end{tabular}

Fuente: Encuesta. 


\section{CIENCIAMATRIA \\ Revista Interdisciplinaria de Humanidades, Educación, Ciencia y Tecnología \\ Año VIII. Vol. VIII. N¹4. Enero - Junio. 2022 \\ Hecho el depósito de ley: pp201602FA4721 \\ ISSN-L: 2542-3029; ISSN: 2610-802X \\ Universidad Nacional Experimental Francisco de Miranda (UNEFM). Santa Ana de Coro. Venezuela}

Luís Antonio Visurraga-Camargo

De los 180 encuestados, según la tabla № 06, un 1.7 \% (3)manifiesta que la supervisión de la gestión en el sector público de la Región Junín es nula (siempre) y un 11.67 \% (21) de los encuestados manifiesta que la supervisión de la gestión es baja (nunca), en tanto que un $17.22 \%$ (31) de los encuestados manifiesta que la supervisión de la gestión es intermedia(casi nunca), en contraste con un $32.22 \%(58)$ y $37.22 \%$ (67) que manifiestan que la supervisión de la gestión en el sector público de Junín, es alta (a veces) y muy alta (casi siempre).

\section{Tabla 7.}

Vigilancia de la Gestión.

\begin{tabular}{llrrrr}
\hline \hline & & & \multicolumn{2}{c}{ Porcentaje } & \multicolumn{2}{c}{$\begin{array}{c}\text { Porcentaje } \\
\text { acumulado }\end{array}$} \\
\hline Válido & Frecuencia & Porcentaje & \multicolumn{2}{c}{ válido } & 10.6 \\
& NUNCA & 19 & 10.6 & 30.6 & 41.1 \\
& A VECES & 55 & 30.6 & 30.6 & 66.7 \\
& CASI SIEMPRE & 46 & 25.6 & 25.6 & 100.0 \\
& SIEMPRE & 60 & 33.3 & 33.3 & \\
& Total & 180 & 100.0 & 100.0 & \\
\hline \hline
\end{tabular}

Fuente: Encuesta.

De los 180 encuestados, según la tabla $N^{\circ} 07$, un 10.56\% (19) manifiesta que la vigilancia de la gestión en el sector público de la Región Junín es nula (siempre) y un 25.56 \% (46) de los encuestados manifiesta que la vigilancia de la gestión es intermedia(casi nunca), en contraste con un $30.56 \%(55)$ y $33.33 \%(60)$ que manifiestan que la vigilancia de la gestión en el sector público de Junín, es alta ( a veces) y muy alta ( siempre). 


\section{CIENCIAMATRIA}

Revista Interdisciplinaria de Humanidades, Educación, Ciencia y Tecnología

Año VIII. Vol. VIII. N¹4. Enero - Junio. 2022

Hecho el depósito de ley: pp201602FA4721

ISSN-L: 2542-3029; ISSN: 2610-802X

Universidad Nacional Experimental Francisco de Miranda (UNEFM). Santa Ana de Coro. Venezuela

Luís Antonio Visurraga-Camargo

\section{Tabla 8.}

Verificación de la Gestión.

\begin{tabular}{llrrrr}
\hline & & & & \multicolumn{2}{c}{ Porcentaje } \\
válido & $\begin{array}{c}\text { Porcentaje } \\
\text { acumulado }\end{array}$ \\
\hline Válido & NUNCA & 19 & 10.6 & 10.6 & 10.6 \\
& CASI NUNCA & 31 & 17.2 & 17.2 & 27.8 \\
& A VECES & 108 & 60.0 & 60.0 & 87.8 \\
& CASI SIEMPRE & 22 & 12.2 & 12.2 & 100.0 \\
& Total & 180 & 100.0 & 100.0 & \\
\hline
\end{tabular}

Fuente: Encuesta.

De los 180 encuestados, según la tabla $N^{\circ}$ 08, un $10.56 \%$ (19) manifiesta que la verificación de la gestión en el sector público de la Región Junín es nula (nunca) y un $12.22 \%$ (22) de los encuestados manifiesta que la verificación de la gestión es baja (casi siempre), y un $17.22 \%$ (31) de los encuestados manifiesta que la verificación de la gestión es intermedia (casi nunca) en contraste con un y $60.00 \%$ ( 108) que manifiestan que la verificación de la gestión en el sector público de Junín, es muy alta (a veces)

\section{Tabla 9.}

Resultados de la Gestión.

\begin{tabular}{llrrrr}
\hline & & & \multicolumn{2}{c}{$\begin{array}{c}\text { Porcentaje } \\
\text { válido }\end{array}$} & $\begin{array}{c}\text { Porcentaje } \\
\text { acumulado }\end{array}$ \\
\hline Válido & NUNCA & 5 & 2.8 & 2.8 & 2.8 \\
& CASI NUNCA & 14 & 7.8 & 7.8 & 10.6 \\
& A VECES & 61 & 33.9 & 33.9 & 44.4 \\
& CASI SIEMPRE & 43 & 23.9 & 23.9 & 68.3 \\
& SIEMPRE & 57 & 31.7 & 31.7 & 100.0 \\
& Total & 180 & 100.0 & 100.0 & \\
\hline \hline
\end{tabular}

Fuente: Encuesta. 


\section{CIENCIAMATRIA \\ Revista Interdisciplinaria de Humanidades, Educación, Ciencia y Tecnología \\ Año VIII. Vol. VIII. №14. Enero - Junio. 2022 \\ Hecho el depósito de ley: pp201602FA4721 \\ ISSN-L: 2542-3029; ISSN: 2610-802X \\ Universidad Nacional Experimental Francisco de Miranda (UNEFM). Santa Ana de Coro. Venezuela}

Luís Antonio Visurraga-Camargo

De los 180 encuestados, según la tabla $N^{\circ} 09$, un $2.78 \%$ (15) manifiesta que los resultados de la gestión en el sector público de la Región Junín es nula (nunca) y un $7.78 \%$ (14) de los encuestados manifiesta que los resultados de la gestión es baja (casi nunca), y un $23.89 \%$ (43) de los encuestados manifiesta que los de la gestión es intermedia(casi siempre) en contraste con un $31.67 \%$ (57) que manifiestan que los resultados de la gestión en el sector público de Junín, es alta (siempre) y finalmente un $33.89 \%$ (61) que manifiestan que los resultados de la gestión en el sector público de Junín, es muy alta (a veces).

\section{CONCLUSIÓN}

Se concluye que existe una relación directa y significativa entre la auditoria de gestión y el control gubernamental en el sector público de la Región Junín. Es recomendable que la Gobernación Regional de Junín, sus autoridades y sus funcionarios del Gobierno Regional, en coordinación con el Órgano de Control Interno, gestionen supervisar, vigilar, verificar los resultados de los Planes Operativos Institucionales de todas las instituciones públicas de la Región Junín, de acuerdo a las directivas y normas aprobadas por la Contraloría General de la República como órgano rector del Sistema Nacional de Control.

\section{FINANCIAMIENTO}

No Monetario.

\section{AGRADECIMIENTO}

A la Universidad Nacional Federico Villarreal, Lima; por motivar el desarrollo de la investigación. 
CIENCIAMATRIA

Revista Interdisciplinaria de Humanidades, Educación, Ciencia y Tecnología

Año VIII. Vol. VIII. N¹4. Enero - Junio. 2022

Hecho el depósito de ley: pp201602FA4721

ISSN-L: 2542-3029; ISSN: 2610-802X

Universidad Nacional Experimental Francisco de Miranda (UNEFM). Santa Ana de Coro. Venezuela

Luís Antonio Visurraga-Camargo

\section{REFERENCIAS CONSULTADAS}

Aroca Sevillano, J. (2016) La auditoría interna y su incidencia en la gestión de empresa de Transportes Guzmán S.A. de la ciudad de Trujillo. [The internal audit and its impact on the management of Transportes Guzmán S.A. in the city of Trujillo] Tesis de maestría. Universidad Nacional de Trujillo. Perú. Recuperado de: https://n9.cl/4yw80

Cabana, S., Cortés, F., Contreras, F., y Vargas, V. (2020). Influencia del control de gestión al valor público generado en servicios dependientes del Ministerio de economía, fomento y turismo, Chile. [Influence of management control on the public value generated in services under the Ministry of Economy, Development and Tourism, Chile] Información tecnológica, 31(2), 103-116. https://dx.doi.org/10.4067/S0718$\underline{07642020000200103}$

Calle Parra, C. (2015) Auditoría de gestión como herramienta de mejoramiento continuo a la Empresa Municipal de Agua Potable, Alcantarillado y Saneamiento Ambiental del cantón Azogues (EMAPAL), periodo 2013. [Management audit as a tool for continuous improvement of the Municipal Company of Drinking Water, Sewerage and Environmental Sanitation of the canton Azogues (EMAPAL), period 2013] Tesis de grado. Universidad Politécnica Salesiana. Ecuador. Recuperado de: https://n9.cl/x3lj7

Congreso de la República. (2002). Ley Nro. 27785. - Ley Orgánica del Sistema Nacional de Control y de La Contraloría General de La República. [Law No. 27785. - Organic Law of the National Control System and the Office of the Comptroller General of the Republic.] Publicada en Diario Oficial el Peruano del 23 de julio de 2002. Perú. Recopilado de: https://n9.cl/m63iz

Flores Tello, C. (2014). Planeamiento y estrategias en la auditoría de gestión en las instituciones públicas del sector energía y minas. [Planning and strategies in the management audit in public institutions in the energy and mining sector] Tesis doctoral. Universidad Nacional Mayor de San Marcos. Lima. Perú. Recuperado de: https://n9.cl/78y7u

Hernández, R., Fernández, C., y Baptista, M. (2014). Metodología de la Investigación [Investigation Methodology] (Quinta Edición ed.). México: McGraw-Hill. 


\section{CIENCIAMATRIA}

Revista Interdisciplinaria de Humanidades, Educación, Ciencia y Tecnología

Año VIII. Vol. VIII. N¹4. Enero - Junio. 2022

Hecho el depósito de ley: pp201602FA4721

ISSN-L: 2542-3029; ISSN: 2610-802X

Universidad Nacional Experimental Francisco de Miranda (UNEFM). Santa Ana de Coro. Venezuela

Luís Antonio Visurraga-Camargo

Madrid Valerio, C., y Palomino Ramírez, W. (2020). Oportunidades de corrupción y pandemia: el compliance gubernamental como un protector eficaz al interior de las organizaciones públicas. [Opportunities for corruption and pandemic: government compliance as an effective protector within public organizations]. Desde el Sur, 12(1), 213-239. https://dx.doi.org/10.21142/des-1201-2020-0014

Portal Martínez, J. (2016). Control interno e integridad: elementos necesarios para la gobernanza pública. [Internal control and integrity: elements necessary for public governance] El Cotidiano, (198), 7-13. Recuperado de: https://n9.cl/ru5os

Santamaría-Quishpe, G., Marcelo-Cárdenas, M., (2017) La Auditoría de Gestión, una Herramienta Necesaria para la Economía. [The Management Audit, a Necessary Tool for the Economy] UT Ciencia Ciencia y Tecnología al servicio del pueblo, 3(2),95-103, Recuperado de: https://n9.cl/un31

Zambrano Zambrano, M. y Armada Trabas, E. (2018). Ciencia y auditoría de gestión en las instituciones públicas de educación superior de la República del Ecuador: Impacto social. [The Management Audit, a Necessary Tool for the Economy] Cofin Habana, 12(1), 15-31. Recuperado de: https://n9.cl/m119e

C2022 por los autores. Este artículo es de acceso abierto y distribuido según los términos y condiciones de la licencia Creative Commons Atribución-NoComercial-Compartirlgual 4.0 Internacional (CC BY-NC-SA 4.0)

(https://creativecommons.org/licenses/by-nc-sa/4.0/). 